Maier, Maja S.

\title{
Erziehungswissenschaftliche Geschlechterforschung? Ein Essay zu Verhältnisbestimmung und Forschungsprogrammatik
}

Baar, Robert [Hrsg.]; Hartmann, Jutta [Hrsg.]; Kampshoff, Marita [Hrsg.]: Geschlechterreflektierte Professionalisierung. Geschlecht und Professionalität in pädagogischen Berufen. Opladen ; Berlin ; Toronto : Verlag Barbara Budrich 2019, S. 15-29. - (Jahrbuch erziehungswissenschaftliche Geschlechterforschung; 15)

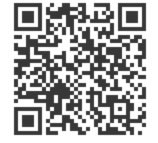

Quellenangabe/ Reference:

Maier, Maja S.: Erziehungswissenschaftliche Geschlechterforschung? Ein Essay zu

Verhältnisbestimmung und Forschungsprogrammatik - In: Baar, Robert [Hrsg.]; Hartmann, Jutta [Hrsg.]; Kampshoff, Marita [Hrsg.]: Geschlechterreflektierte Professionalisierung. Geschlecht und

Professionalität in pädagogischen Berufen. Opladen ; Berlin ; Toronto : Verlag Barbara Budrich 2019, S. 15-29 - URN: urn:nbn:de:0111-pedocs-219011 - DOI: 10.25656/01:21901

https://nbn-resolving.org/urn:nbn:de:0111-pedocs-219011

https://doi.org/10.25656/01:21901

in Kooperation mit / in cooperation with:

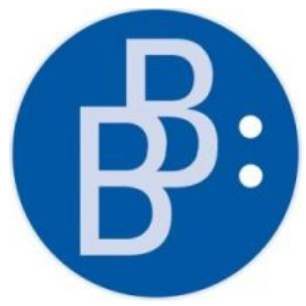

https://www.budrich.de

\section{Nutzungsbedingungen}

Gewährt wird ein nicht exklusives, nicht übertragbares, persönliches und beschränktes Recht auf Nutzung dieses Dokuments. Dieses Dokument ist ausschließlich für den persönlichen, nicht-kommerziellen Gebrauch bestimmt. Die Nutzung stellt keine Übertragung des Eigentumsrechts an diesem Dokument dar und gilt vorbehaltlich der folgenden Einschränkungen Auf sämtlichen Kopien dieses Dokuments müssen alle Urheberrechtshinweise und sonstigen Hinweise auf gesetzlichen Schutz beibehalten werden. Sie dürfen dieses Dokument nicht in irgendeiner Weise abändern, noch dürfen Sie dieses Dokument für öffentliche oder kommerzielle Zwecke vervielfältigen, öffentlich ausstellen, aufführen, vertreiben oder anderweitig nutzen.

Mit der Verwendung dieses Dokuments erkennen Sie die Nutzungsbedingungen an.

\section{Terms of use}

We grant a non-exclusive, non-transferable, individual and limited right to using this document.

This document is solely intended for your personal, non-commercial use. Use of this document does not include any transfer of property rights and it is conditional to the following limitations: All of the copies of this documents must retain all copyright information and other information regarding legal protection. You are not allowed to alter this document in any way, to copy it for public or commercial purposes, to exhibit the document in public, to perform, distribute or otherwise use the document in public.

By using this particular document, you accept the above-stated conditions of use.

\section{Kontakt / Contact:}

\section{peDOCs}

DIPF | Leibniz-Institut für Bildungsforschung und Bildungsinformation Informationszentrum (IZ) Bildung

E-Mail: pedocs@dipf.de

Internet: www.pedocs.de

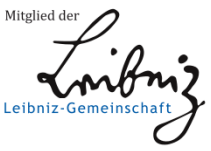


Robert Baar

Jutta Hartmann

Marita Kampshoff (Hrsg.)

\section{$15 / 2019$}

Jahrbuch erziehungswissenschaftliche Geschlechterforschung

Geschlechterreflektierte Professionalisierung Geschlecht und Professionalität in pädagogischen Berufen 


\section{Robert Baar}

Jutta Hartmann

Marita Kampshoff (Hrsg.)

\section{Geschlechterreflektierte Professionalisierung - Geschlecht und Professionalität in pädagogischen Berufen}

Verlag Barbara Budrich Opladen • Berlin • Toronto 2019 
Bibliografische Information der Deutschen Nationalbibliothek

Die Deutsche Nationalbibliothek verzeichnet diese Publikation in der Deutschen

Nationalbibliografie; detaillierte bibliografische Daten sind im Internet über

http://dnb.d-nb.de abrufbar.

Gedruckt auf säurefreiem und alterungsbeständigem Papier.

Alle Rechte vorbehalten.

(C) 2019 Verlag Barbara Budrich, Opladen, Berlin \& Toronto

www.budrich-verlag.de

ISBN 978-3-8474-2277-8 (Paperback)

eISBN 978-3-8474-1310-3 (eBook)

Das Werk einschließlich aller seiner Teile ist urheberrechtlich geschützt. Jede Verwertung außerhalb der engen Grenzen des Urheberrechtsgesetzes ist ohne Zustimmung des Verlages unzulässig und strafbar. Das gilt insbesondere für Vervielfältigungen, Übersetzungen, Mikroverfilmungen und die Einspeicherung und Verarbeitung in elektronischen Systemen.

Typographisches Lektorat: Anja Borkam, Jena

Umschlaggestaltung: disegno visuelle kommunikation, Wuppertal - www.disenjo.de Druck: paper \& tinta, Warschau

Printed in Europe 


\section{Inhalt}

\section{Einleitung}

Robert Baar, Jutta Hartmann \& Marita Kampshoff

Vorwort. 7

Robert Baar, Jürgen Budde, Marita Kampshoff \& Astrid Messerschmidt Redaktion des Jahrbuchs erziehungswissenschaftliche

Geschlechterforschung

Von der Frauen- und Geschlechterforschung in der

Erziehungswissenschaft zur erziehungswissenschaftlichen

Geschlechterforschung....

Maja S. Maier

Erziehungswissenschaftliche Geschlechterforschung?

Ein Essay zu Verhältnisbestimmung und Forschungsprogrammatik 15

Robert Baar, Jutta Hartmann \& Marita Kampshoff

Geschlechterreflektierte Professionalisierung - Geschlecht und

Professionalität in pädagogischen Berufen. Eine Einführung

\section{Vielfältige Lebensweisen im Fokus von Professionalität}

Florian Cristobal Klenk

Interdependente Geschlechtervielfalt als un/be/deutende Anforderung an pädagogische Professionalität

Mart Busche \& Uli Streib-Brzič

Die Entwicklung heteronormativitätskritischer Professionalität in

Reflexions-Workshops - Zur Verbindung von pädagogischem

Erfahrungswissen und wissenschaftlichem Erkenntniswissen im Kontext

von Praxisforschung

Carolin Vierneisel \& Johannes Nitschke

(De-)Professionalisierungstendenzen?! Vielfalts*sensible Bildung im

Lehramtsstudium. 


\section{Geschlechterreflektierte Professionalität im Elementarbereich}

\section{Melanie Kubandt}

Ansprüche an ein geschlechtergerechtes, professionelles Handeln im Elementarbereich - Ethnographische Perspektiven auf Ungewissheiten,

Komplexitäten und Grenzen im pädagogischen Alltag

Susann Fegter, Anna Hontschik, Eszter Kadar, Kim-Patrick Sabla \& Maxine Saborowski

Bezüge auf Familie als Moment der Vergeschlechtlichung pädagogischer Professionalität: Diskursanalytische Perspektiven auf Äußerungen in Gruppendiskussionen mit Kita-Teams

\section{Herausforderungen sexualpädagogischer Professionalisierung}

\section{Anja Eichhorn}

Doing Sexual Agency: Sexuelle Handlungsfähigkeit sexuell

missbrauchter jugendlicher Mädchen in der stationären Jugendhilfe

Marion Thuswald

Geschlechterreflektierte sexuelle Bildung?

Heteronormativität und Verletzbarkeit als Herausforderungen

sexualpädagogischer Professionalisierung

\section{Rezension}

Marina Dangelat, Frauke Grenz \& Christine Thon

Rezension zu: Gesicht Zeigen! (2017) (Hrsg.): „Weiße können nicht rappen“. Das Positionierungsspiel gegen Vorurteile und Klischees und zu: Wedl, Juliette (2018): Identitätenlotto. Ein Spiel quer durchs Leben ... 183

Verzeichnis der Autor_innen 191 
Maja S. Maier

\section{Erziehungswissenschaftliche Geschlechterforschung? Ein Essay zu Verhältnisbestimmung und Forschungsprogrammatik}

\section{Einige Vorbemerkungen}

$\mathrm{Zu}$ behaupten, dass Gender Studies an Aktualität ${ }^{1}$ verloren haben und die Erforschung gesellschaftlicher Differenzierungsprozesse - insbesondere solcher, die die Felder Erziehung und Bildung tangieren - im Hinblick auf ihre geschlechterdifferierende und hierarchisierende Bedeutung verzichtbar ist, wäre nur im Angesicht einer Gesellschaft möglich, in der der Kategorie Geschlecht für die Strukturierung der sozialen, ökonomischen und reproduktiven - und auch pädagogischen - Ordnung keinerlei Erklärungskraft mehr zukäme. Sich mit Gender Studies zu beschäftigen, erzwingt daher neben der wissenschaftlichen immer auch eine politische Positionierung, die sich nach ,außen', aber auch nach ,innen' richtet: Schließlich ist im Anschluss an die machtanalytische feministische Wissenschaftskritik (u. a. Haraway 1996) immer zu fragen, wie die Perspektiven und Positionen von gesellschaftlichen Gruppen und wissenschaftlichen Diskursgemeinschaften wie der postkolonialen Kritik, Critical Whiteness Studies, Disability Studies und queertheoretischen, anti-kategorialen Ansätzen in die Gender Studies eingehen und wie die Verwobenheit der eigenen Wissenschaftspraxis in gesellschaftliche und globale Machtverhältnisse und Herrschaftsdiskurse kritisch reflektiert werden kann. Denn Gender Studies priorisieren , gender' mit allen Implikationen und Kontroversen als legitimen Leitbegriff wissenschaftlicher Analyse und behaupten ihn gegenüber anderen Leitbegriffen wie Diversity, Intersektionalität, Inklusion oder auch Queer.

Das Verhältnis der Erziehungswissenschaft zu interdisziplinären Forschungsfeldern wird derzeit zwar nicht für die Gender Studies, aber für die empirische Bildungsforschung diskutiert (Koller 2018). Deren Konjunktur

1 Diese Frage bezieht sich auf die Anfrage für einen wissenschaftlichen Vortrag, auf dessen Manuskript sich der vorliegende Beitrag stützt. In der Frage symbolisiert sich schon die Position der Gender Studies zwischen Wissenschaft, Öffentlichkeit und Politik, die hier zum Gegenstand einer analytischen Betrachtung gemacht wird.

https://doi.org/10.3224/jeg.v15i1.02 
lässt die Frage zentral werden, wie Bildung und Erziehung zum Gegenstand von empirischer Forschung gemacht werden können, wo sie doch selbst ebenso wie die Theorien über sie von normativ begründeten Vorannahmen durchdrungen sind. Dass Erziehung und Bildung vom Zusammenspiel gesellschaftlicher Erwartungen, normativer Bestimmungen und pädagogischer Praxis konturiert sind, stellt die empirisch-erziehungswissenschaftliche Erkenntnisproduktion folglich vor ein gravierendes Forschungsproblem, das im Rahmen erziehungswissenschaftlicher Reflexion auch zunehmend Aufmerksamkeit erhält (Dinkelaker et al. 2016: 13). Die folgenden Überlegungen setzen an der hier aufgeworfenen Frage an, ob und wie sich der Gegenstand einer sich als erziehungswissenschaftlich verstehenden Forschung bestimmen lässt und nehmen dazu das Verhältnis von Erziehungswissenschaft und Frauen- und Geschlechterforschung als einem interdisziplinären Feld in den Blick, das sich von Beginn an stark über empirische Forschung legitimiert und etabliert hat.

Verweisen Frauen- und Geschlechterforschung oder auch Gender Studies schon dem Begriff nach auf wissenschaftliche Kontroversen über theoretische Annahmen, methodische Zugänge und untersuchte Gegenstände und ihre Dynamik, suggeriert die Rede von der Erziehungswissenschaft ${ }^{2}$ die Annahme einer an einer einheitlich verstandenen Kernproblemantik ausgerichteten Disziplin. Ob die Erziehungswissenschaft aber eher als Handlungswissenschaft bzw. handlungsleitende Disziplin oder wie andere Disziplinen ausschließlich als Reflexionswissenschaft verstanden wird - was jeweils folgenreich für die Bestimmung einer solchen Kernproblematik wäre - bleibt jedoch innerhalb der Disziplin strittig (schon früh dazu König/Zedler 1983; jüngst z.B. Vogel 2016). Ohne sich in disziplinpolitischen Diskussionen zu verlieren, lässt sich für den vorliegenden Zusammenhang konstatieren, dass es gerade die Heterogenität an Theorien, Methoden und Praxisbezügen ist, die das disziplinäre Selbstverständnis ausmachen (vgl. dazu aktuell z.B. Terhart 2016) und dass diese zudem dynamisch und fluide sein kann (Terhart 2012: 17f.). ${ }^{3}$ Wie nun vor diesem Hintergrund das Verhältnis von dynamisch-fluiden und kontrovers diskutierten wissenschaftlichen Disziplinen und politischer Öffentlichkeit analytisch zu fassen ist, wird im Folgenden entlang eines wissenschaftstheoretisch informierten Blicks auf die Dynamiken der Gender Studies und der Erziehungswissenschaft dargelegt. Dafür werden in einer kursorischen Re-Lektüre zentrale Diskurslinien aufgegriffen und skizzenhaft entfaltet, wie eine erziehungswissenschaftlich konturierte Geschlechterforschung begründet werden könnte.

2 Zur Benennung erziehungswissenschaftlicher Institute vgl. Terhart (2012) und Studiengänge vgl. Grunert/Ludwig (2016).

3 Zur Aktualität des „Ringens“ mit disziplinärer Heterogenität am Beispiel der Studiengangsgestaltung vgl. Ludwig (2018). 


\section{Gender Studies zwischen Wissenschaftsdisziplin und Politik}

Betrachtet man das Selbstverständnis der Gender Studies, lassen sich zwei Pole der Diskussion unterscheiden: So hob Susanne Völker als Sprecherin der deutschsprachigen Fachgesellschaften für Geschlechterforschung/-studien 2017 unter dem Titel „Aktuelle Herausforderungen der Geschlechterforschung" zur Eröffnung der gleichnamigen Konferenz auf drei Punkte ab, die die Agenda der Gender Studies bestimmen (sollten): Erstens hätten Gender Studies im Anschluss an die feministische Wissenschaftskritik die Aufgabe, die wirklichkeitsschaffende wissenschaftliche Praxis vor dem Hintergrund der globalen Verschiebungen von Macht- und Hierarchieverhältnissen zu reflektieren. Im Anschluss an die Arbeiten von Judith Butler sei zweitens das Sichtbar-werden-lassen von Identitäten notwendig, die von machtwirksamen Differenzsetzungen auf soziale Unorte verwiesen werden und bleiben. Drittens sei es aufgrund der jüngeren Entwicklungen in der Landschaft politischer Parteien - nicht nur hinsichtlich der Bedeutung von Geschlecht - unerlässlich, die dominanten Differenzsetzungen im öffentlichen und politischen Diskurs und das, worauf sie reagieren, zu rekonstruieren. In dieser programmatischen Standortbestimmung tritt der mit ihren feministischen Traditionen verbundene kritische und mitunter auch selbstkritische Impetus der Gender Studies hervor, mit dem ihre Aktualität als transdisziplinär ausgerichtete Wissenschaftsdisziplin, aber auch als politisch-ethisches Projekt begründet wird (Völker 2017).

Im Unterschied dazu hat Stefan Hirschauer, seinerseits Repräsentant soziologischer Geschlechterforschung, 2014 recht polemisch dafür plädiert, Gender Studies nur mehr als wissenschaftliche Disziplin auszurichten, sie als übergreifende Differenzierungsforschung zu rahmen und daran zu arbeiten, dass sie endlich in den Kanon der einzelnen Fächer eingearbeitet werden. Zwingend ist dafür aus seiner Perspektive eben gerade die Entpolitisierung der Geschlechterforschung im Zuge einer Ablösung von ihrer identitätspolitisch aufgeladenen Tradition (Hirschauer 2014: 882).

An den beiden Polen wird die Kontroverse deutlich, die die Gender Studies nach der Abkehr von den Ursprüngen der parteilichen Frauenforschung vielfach und immer wieder neu durchzieht. Diese rankt sich nicht zuletzt um die Frage: Welchen Stellenwert sollen soziale Bewegungen, ihre identitätspolitischen Fundierungen und politischen Forderungen für Gender Studies als Wissenschaftsdisziplin haben? Diese Frage stellt sich dabei nicht nur für die Gender Studies, sondern auch für die Erziehungswissenschaft. So hat Juliane Jacoby die Verhältnisbestimmung von sozialer Bewegung und wissenschaftlicher Disziplin 2008 als eine besonders komplexe Herausforderung markiert: 
„Ihre [hier: Erziehungswissenschaft] Konstitution durch diesen Gegenstand [hier: Erziehung] macht sie anfällig für das Versprechen, gesellschaftsveränderndes Potenzial zu generieren. Ihre im Gegenstand liegende Nähe zur Ethik verkompliziert den Zugang zur Empirie. Weil sie immer den Spagat zwischen analytisch und methodisch abgesicherter Forschung und normativen Entscheidungen machen muss, ist es für die Erziehungswissenschaftlerinnen besonders notwendig, aber wohl auch besonders schwierig, die wissenschaftstheoretische Frage, wie man das Verhältnis von sozialer Bewegung und Wissenschaft definiert, zu beantworten.“ (Jacobi 2008: 85)

Gleichwohl diese Frage kaum abschließend beantwortet werden kann, zeugt die Institutionalisierungsgeschichte der Gender Studies in der Erziehungswissenschaft davon, dass sie diesen Spagat - um in der Metapher zu bleiben zumindest sportlich gemeistert hat und weiterhin meistert. Die Gründungsgeschichte der Sektion, die aus ihr hervorgehenden Schriftenreihen und die strukturelle Verankerung von sog. , Geschlechterthemen' in Curricula und Studiengänge sowie eine überaus lebendige Forschungs-, Diskurs- und Publikationslandschaft lassen sich als Erfolgsgeschichte beschreiben - auch wenn die Etablierung geschlechterrelevanter Aspekte in der Geschichtsschreibung der Disziplin bislang nur partiell erreicht wurde. Hierzu müsste die Disziplingeschichte der Erziehungswissenschaft einer machtkritischen Analyse unterzogen werden, um schließlich - wie es Rieger-Ladich im Rückgriff auf Foucaults „Ordnung des Diskurses“ vorgeschlagen hat - herausarbeiten, ,[...] was innerhalb der EW welchen Fachvertreter/innen als wahrheitsfähig gilt (und was nicht), was von welchen Gruppen als ernstzunehmendes Forschungsthema eingeschätzt wird (und was nicht), was von den Autorisierungsinstanzen zum kanonischen Wissensbestand der Disziplin gezählt wird (und was nicht). " (Rieger-Ladich 2009: 18) Die hier benannte disziplin- bzw. wissenschaftshistorische Leerstelle setzt sich im Feld der systematischen Erziehungswissenschaft fort: Nach wie vor finden sich im allgemein-erziehungswissenschaftlichen Kanon kaum geschlechtertheoretisch fundierte Bearbeitungsformen ihrer Grundfragen. Es fehlt, wie bereits 1991 im Antrag auf Einrichtung einer Kommission Frauenforschung in der Deutschen Gesellschaft für Erziehungswissenschaft erwähnt, eine auf geschlechtertheoretische Arbeiten zurückgehende „Erweiterung und Differenzierung bisheriger erziehungswissenschaftlicher Denkmodelle“ (AG Frauenforschung 1991: 2). ${ }^{4}$

Die politische Sprengkraft der anfänglich parteilichen pädagogischen Frauenforschung mag also mit ihrer akademischen Institutionalisierung - gemäß der marx'schen Lesart - geringer geworden sein. Ihre wissenschaftliche, also theoretische, methodologische und methodische Entwicklung hat jedoch davon profitiert, nicht zuletzt auch durch immer wieder neue politische Forderungen der unterschiedlichen sozialen Bewegungen: Was es genau meint,

Gleichwohl gingen aus der Frauen- und Geschlechterforschung immer wieder wichtige Impulse für die sozial- und erziehungswissenschaftliche Theoriebildung und Methodenentwicklung hervor. 
wenn auf der Homepage der Sektion Frauen- und Geschlechterforschung der DGfE steht, dass hier Wissenschaftler*innen ,,aus einer Geschlechterperspektive an Fragestellungen zu Theorie und Praxis pädagogischen Denkens und Handelns arbeiten", bleibt dabei erfrischend ungefasst. Dass hier kein grundlegenderes gemeinsames Programm formuliert wird und Benennungen (der Sektion und ihrer Organe) immer wieder neu diskutiert werden, zeugt nicht zuletzt von einer weiteren besonderen Schwierigkeit der erziehungswissenschaftlichen Geschlechterforschung: Der entscheidenden Frage, ob denn nun Geschlechterfragen als problemorientierte und/oder als disziplinorientierte Themenbereiche bearbeitet werden soll(t)en - so 2013 aufgeworfen von Klinger im Rundbrief der Sektion (vgl. Vorstand der Sektion FuG in der DGfE 2013: 27).

Den bis vor kurzem noch im Netz zugänglichen Sitzungsprotokollen der Sektion lässt sich entnehmen, dass aus einer 1982 initiierten und 1984 institutionalisierten Arbeitsgruppe 1991 die Kommission für Frauenforschung in der Erziehungswissenschaft wurde, die bis zum Sektionsstatus 1999 auch diese Bezeichnung führte. Gegen die alternativen Bezeichnungen „Geschlechterforschung“, „Feministische Erziehungswissenschaft" und „Frauenforschung“ entschieden sich die Mitglieder dann mehrheitlich für die Bezeichnung „Frauen- und Geschlechterforschung“, wobei das Stimmenverhältnis bei der Stichwahl zwischen „Frauen- und Geschlechterforschung“ und „Frauenforschung“ 18:12 betrug (vgl. Sektion FuG o.J.). Dieses knappe Ergebnis zeugt einerseits von deutlichen wissenschaftlichen Differenzen in Theoriebezügen und Gegenstandsbestimmungen; andererseits drückt sich darin aber insbesondere die Kontroverse darüber aus, ob und wie solche Differenzen, die dynamischen wissenschaftlichen Feldern immanent sind, im Selbstverständnis integriert werden können oder aber in programmatischer Absicht symbolisch markiert werden sollen. ${ }^{5}$ Mit der Entscheidung zu Frauen- und Geschlechterforschung in der Erziehungswissenschaft wurde seither jedenfalls auf eine engere disziplinäre Anbindung verzichtet.

Eine deutliche Disziplinorientierung und eine ebenso deutliche Anbindung an die emanzipatorische Frauenbewegung dokumentiert demgegenüber die erste 6-bändige Schriftenreihe mit dem Titel „Einführung in die pädagogische Frauenforschung" - gleichwohl bereits hierin nicht nur von Frauen die Rede war. Unter dem Titel „Jahrbuch Frauen- und Geschlechterforschung in der Erziehungswissenschaft" verbreitert sich Jahre später das Spektrum der Beiträge in geschlechtertheoretischer und in disziplinärer Hinsicht. Abgesteckt wird ein Feld, das von geschlechtertheoretischen Ansätzen, kategoriengeleiteten For-

5 Umbenennungen eines wissenschaftlichen Feldes verweisen auf Paradigmenwechsel und neue symbolische Grenzziehungen; sie können auch von sozialen und generationalen Bewegungen beeinflusst sein. Eine historisch-empirische Rekonstruktion der Grenzziehungspraktiken der Gender Studies wäre insofern interessant. 
schungsperspektiven und geschlechterpolitischen Themen (Stichwort: Differenz, Diversity, Queer) zu Themen wie Sexualität, Migration, Sozialisation, Care, Inklusion reicht, die im engeren Sinne als pädagogisch relevant bezeichnet werden können. An dieser Unterscheidung wird schon deutlich, dass es alles andere als einfach ist, genuin erziehungswissenschaftliche Themen oder Fragestellungen trennscharf zu benennen. So werden im Rahmen der erziehungswissenschaftlichen Gender Studies Fragestellungen zu Bildungsbiografien, Strukturen des Bildungssystems, Bildungsungleichheit, Bildungs- und Subjekttheorien, Sozialisation, Körper und Identität, Kindheit und Jugend, Lebensphasen oder zu pädagogischer Professionalität bearbeitet und Bezüge zu den gesellschaftlichen Bedingungen hergestellt, in denen sie gedacht werden, wie etwa der Globalisierung, der neoliberalen Transformation gesellschaftlicher Funktionssysteme oder der Migrationsgesellschaft.

\section{Erziehungswissenschaftliche Forschung?}

Die Frage, was den Kern der erziehungswissenschaftlichen Problemstellungen und die Wege der Erkenntnisproduktion kennzeichnet, begleitet die Disziplin seit Beginn ihrer Institutionalisierung. Die Erziehungswissenschaft ist dabei mittlerweile nicht mehr nur dem Spannungsfeld ausgesetzt, das sich klassisch als Theorie-Praxis-Problem konstruiert (früh dazu Benner 1980) - sondern zunehmend auch einer veränderten Verhältnisbestimmung von wissenschaftlicher Analyse und Nutzenerwartung: Die Diskussion um eine evidenzbasierte Erziehungswissenschaft, deren maximale Differenz zu anderen erziehungswissenschaftlichen Richtungen sich in der Produktion von Steuerungswissen und Politikberatung ausdrückt, wird mittlerweile auch innerdisziplinär - in teilweise kritischer Absicht - geführt (z.B. Tillmann/ Baumert 2016). Denn die Erziehungswissenschaft wird mit Forschungsanforderungen konfrontiert, die zumindest aus disziplinhistorischer Perspektive betrachtet - die Konflikte zwischen empirischer, geisteswissenschaftlicher und kritischer Erziehungswissenschaft erneut aufrütteln. So lassen neu entstehende Organisationsformen, wie die 2012 ausgegründete Gesellschaft für empirische Bildungsforschung, er-

6 Ganz aktuell bestimmt die Bundesministerin für Bildung und Forschung das Verhältnis von Forschung und Praxis als reziprok unmittelbar verknüpft: „Die Bildungsforschung schafft Wissen und damit Grundlagen für Empfehlungen, die wir Politiker genauso benötigen wie Erzieher, Lehrer und Professoren. Und auch umgekehrt gilt: Praxisrelevante Forschung benötigt Wissen aus dem pädagogischen Alltag, um die ,richtigen“, die relevanten Forschungsfragen entwickeln zu können." (https://www.bildungsforschungstagung.de/) 
kennen, dass es nicht mehr zu gelingen scheint, konträre Positionen im Verständnis von Bildung und Erziehung in der Disziplin zu integrieren (Helsper 2016).

Die zunehmende Priorisierung empirischer Forschung führt dabei zu zweierlei: Erstens gerät das Verhältnis der erziehungswissenschaftlichen Teildisziplinen in Bewegung. Die allgemeine und die historische Erziehungswissenschaft und vornehmlich theoretisch systematische und hermeneutische Erkenntnisverfahren nutzen, drohen an Bedeutung und Sichtbarkeit zu verlieren (Zedler 2016; Schmid 2008); zweitens erhalten die in der empirischen Forschung starken Bezugsdisziplinen der Erziehungswissenschaft Psychologie und Soziologie in ihren Gegenstandskonstruktionen und Forschungsparadigmen Zuwachs: einerseits von der als psychologischer Lehr-Lernforschung ausgerichtete sog. empirische Bildungsforschung; andererseits von der eng an sozialwissenschaftliche Methodologie orientierten qualitativen Bildungsforschung. Ein Rekurs auf im engeren Sinne erziehungswissenschaftliche Fragestellungen ist dabei in beiden Forschungslinien kaum mehr erkennbar. Dazu hatte Pia Schmid schon 2008 angemerkt, dass die Heinrich Roth zugeschriebene empirische Wendung dazu geführt habe, dass sich die Erziehungswissenschaft als kritische Sozialwissenschaft definiere - und historische und philosophische Erkenntnismethoden demgegenüber an Stellenwert verloren haben (Schmid 2008). Problematisiert wurden die Folgen einer stark sozialwissenschaftlichen Ausrichtung auch schon 1980 von Klaus Mollenhauer, der selbst zugleich als ein Akteur ihrer Etablierung gelten kann. In der Fokussierung auf sozialwissenschaftliche Bezugstheorien und der zunehmenden Methodisierung sah er ein ,Ausweichmanöver' gegenüber den disziplinären Aufgaben. Mit dem Fokus auf die gesellschaftliche Einbettung und Funktion von Bildung und Erziehung waren erziehungswissenschaftliche Fragen, die weder von der Soziologie noch von der Psychologie hinreichend geklärt werden können, aus dem Blick geraten: Das sind zum einen Fragen nach der normativen Ausrichtung und der Legitimierung pädagogischen Handelns und wie diese sich beispielsweise im Kontext von Organisationen, der strukturellen Asymmetrie pädagogischer Beziehungen, sich verändernder gesellschaftlicher Vorstellungen von Bildung und Erziehung, und nicht zuletzt des sich veränderten Generationenverhältnisses konkretisieren. Zum anderen sind das methodologische Überlegungen und Reflexionen dazu, wie zu diesen Fragen ein empirischer Zugang eröffnet werden könnte, wie also beispielsweise mit qualitativen Forschungsmethoden eine - so verstanden - erziehungswissenschaftliche Fragestellung bearbeitet werden kann. ${ }^{7}$ Wie sich eine mögliche grundlagentheoretische erziehungswissenschaftliche Perspektive konkretisieren lassen könnte, wird in jün-

7 Mittlerweile liegen erste bildungstheoretisch fundierte methodologische Weiterentwicklungen für eine erziehungswissenschaftliche Bildungsforschung vor (z.B. Koller/Wulftange 2014; Rosenberg 2011; Müller/Miethe 2012). 
gerer Zeit von in der Tradition der kritischen Erziehungswissenschaft stehenden Perspektiven daher wieder stärker aufgegriffen; beispielsweise in der Diskussion darum, welchen Beitrag die Erziehungswissenschaft zur empirischen Bildungsforschung leistet (Koller 2018) oder auch welche Fragestellungen und Forschungsinteressen die erziehungswissenschaftliche Perspektive in interdisziplinäre Forschungsfelder einbringt (z.B. zu Familie Matthes 2018, zu Diskriminierung Heinemann/Mecheril 2016 oder zu Jugend Pfaff 2015).

\section{Erziehungswissenschaft und Geschlechterforschung - gemeinsame Herausforderungen}

Bevor hier eine mögliche Perspektive für eine erziehungswissenschaftlich-empirische Forschung im Feld von Gender Studies entfaltet wird, sollen die Gemeinsamkeiten, methodologischen Herausforderungen und offenen Fragen einer erziehungswissenschaftlichen Geschlechterforschung skizziert werden: Aus einer wissenschaftstheoretischen Perspektive weisen Gender Studies und Erziehungswissenschaft Ähnlichkeiten auf, da sie beide von einem spezifischen Legitimationsdruck begleitet werden, teils latent, teils explizit Normativität in den Forschungsgegenstand eingeschrieben haben und dafür anfällig sind, direkten Einfluss auf die gesellschaftliche Praxis nehmen zu wollen - oder diesen zugeschrieben zu bekommen. Letzterem stehen beide ambivalent bzw. different gegenüber, denn sie können für jegliche gesellschaftliche Entwicklung, die als Fehlentwicklung oder Forschungsdesiderat betrachtet wird, adressiert oder sogar responsibilisiert werden. Infolge der veränderten externen Ansprüche an und dem veränderten Verständnis von Wissenschaft haben sie auch beide tendenziell einen Reputationsverlust zu verschmerzen. ${ }^{8}$

Denn schließlich wurden zwar die spezifischen methodologischen und methodischen Herausforderungen der erziehungswissenschaftlichen Forschung zu Geschlechterdifferenz und anderen Differenzlinien intensiv bearbeitet (z.B. Buchen et al. 2004; Walgenbach 2014; Emmerich/Hormel 2013). Daraus resultieren jedoch keine, fertigen' Forschungsperspektiven oder anwendungsbezogene Erkenntnisse, sondern ,nur' methodologische und theoretische Reflexionsangebote, die es ermöglichen, die Reifizierungsproblematik im Forschungsprozess immer wieder neu zu bearbeiten. Es bleibt insofern eine Daueraufgabe intersektional angelegter Studien im Feld der Ungleichheits- und

8 Dass dies weit über das Fach hinaus gravierende Folgen haben kann, zeigte der Ungarische Regierungserlass vom 12.10.2018, in dem mit der Begründung, es gebe keine Nachfrage nach den Absolventen der Masterstudiengänge Gender Studies, diese nicht mehr zugelassen werden. 
Diskriminierungsforschung reflexiv einzuholen, dass sie zwar Erkenntnisse über die Wirkmächtigkeit und Wechselwirkungen unterschiedlicher sozialer Kategorien sowie ihrer Herstellung gewinnen, aber zugleich Gefahr laufen, soziale Kategorien in Anschlag und dadurch im Forschungsprozess erst hervorzubringen, zu hierarchisieren und zu stereotypisieren. Eine besondere Schwierigkeit liegt dabei genau in der Verknüpfung der empirischen Befunde und Erkenntnisse mit erziehungswissenschaftlichen Fragestellungen und pädagogischer Praxis: Wird beispielsweise der Einfluss sozialer Kategorien auf pädagogisches Handeln anhand von Forschungsmaterial sichtbar gemacht, führt dies allzu oft erst zur Herausbildung stereotyper Beobachtungs- und Klassifikationsmuster. Wo aber die Auseinandersetzung mit Forschung zur Trivialisierung der Befunde führt, bildet sich ,Herrschaftswissen ' und neue Sagbarkeiten werden erzeugt (wie z.B. ,Inklusionskind“). Die Reifizierungsproblematik reicht insofern über den Forschungsprozess hinaus in den Bereich der Vermittlung - sei es in der Hochschullehre, der Praxis oder im wissenschaftlichen Kontext selbst.

Eine letzte theoretisch-methodologische Herausforderung liegt im Umgang mit den innerdisziplinär durchaus konkurrierenden kategorialen Bezeichnungen: Wie empirische Forschung zwischen kategorialer Identitätspolitik, Analysekategorien und Identitäts- und Gesellschaftskritik konzipiert werden kann, ist nicht einfach zu beantworten. War zunächst die Identitätskategorie ,Frau“ von der Analysekategorie ,gender' abgelöst worden, werden nun im Kontext von LGBTI mit ,all gender', , all sexes' und Sternchen wieder Identitätskategorien in den wissenschaftlichen Diskurs eingebracht - auch wenn diese nicht auf binäre Vorstellungen von Geschlecht, sondern auf dessen Pluralisierung, Fluidität und Diffusion fokussieren. Die wiederkehrenden Modifikationen der Bezugskategorien von Gender Studies bergen insofern für Forschungsanliegen immer wieder neuen Klärungsbedarf.

\section{Programmatische Überlegungen zu einer erziehungswissenschaftlichen Geschlechterforschung}

,Doppelte Historizität‘ ist der Begriff, mit dem die Herausforderungen der Gender Studies vielleicht am besten gerahmt werden können (für Geschlechterbeziehungen und Geschlechterordnungen vgl. Opitz 2008: 13; für Sexualität Baader 2016: 13). Nicht nur die Forschungsgegenstände der Gender Studies sind historisch kontextuiert und somit wandelbar, auch die Forschenden selbst sind eingebunden in die konjunktiven Erfahrungen ihrer Generation und somit 
in ihren Perspektiven und Deutungen immer schon historisch, geformt '. Darauf hat vor allem Karl Mannheim (1928/1929) in seinen wissenssoziologischen Arbeiten aufmerksam gemacht. Wenn man diesen Gedanken nicht ,nur ${ }^{\text {‘ }}$ in eine Forschungsmethode übersetzt, wie das beispielsweise in der Ausarbeitung der Dokumentarischen Methode der Fall ist (z.B. Bohnsack et al. 2001), sondern an die erziehungswissenschaftliche Grundfrage der Generationendifferenz anschließt, dann lässt sich eine Perspektive - wenn auch nur in groben Linien - entfalten, die für die Gender Studies in der Erziehungswissenschaft interessant sein könnte:

Die Generationendifferenz konstituiert mit ihrer davon abgeleiteten Unterscheidung von Kindern, Jugendlichen und Erwachsenen die pädagogische Interaktion und die Asymmetrie der Beziehung. ${ }^{9}$ Sie repräsentiert zugleich die zukunftsbezogene Facette von Bildungsideen und Erziehungstheorien und lässt nicht nur fraglich werden, was „die alte Generation mit der jungen will“ wie Schleiermacher das pointiert hat, sondern sie dient im vorliegenden $\mathrm{Zu}-$ sammenhang dazu, eine programmatische Perspektive für die erziehungswissenschaftliche Geschlechterforschung zu begründen. Konstatieren lässt sich zunächst allerdings seit den 2000er Jahren eine Vernachlässigung der für Bildungs- und Erziehungsprozesse konstitutiven Generationenfrage. ${ }^{10}$

Gerade für die Forschung im Kontext der Gender Studies könnte jedoch die Generationendifferenz eine bedeutsame Rolle spielen, weil sie es im besten Fall vermag, das geschlechterbezogene Selbstverständnis jüngerer Generationen in seiner Bezugnahme zu erfassen: einerseits auf die im Zuge gesellschaftlicher Modernisierung veränderten Geschlechterordnungen, Geschlechterbeziehungen und Geschlechterdiskurse und andererseits auf die geschlechterbezogenen Adressierungen seitens der Erziehendengeneration. Theoretisch könnte dieses Verhältnis schließlich mit dem Goffman'schen Begriff der ,,institutionellen Reflexivität" (Goffman 1994) gefasst werden: Hervorgehoben werden damit die institutionellen Mechanismen, die dazu beitragen, dass die Relevanz der Geschlechterdifferenz in spezifischen sozialen Arrangements derart gespiegelt wird, dass auf diese Weise ein Maß an Glaubwürdigkeit ihrer Sinnhaftigkeit und an Legitimität ihrer Geltung erzeugt wird, dass die Individuen ihr Verhalten, ihre Deutungen und ihre Performances danach ausrichten. Diese Plausibilitätsfiguren - das ist die diesen Überlegungen zugrundeliegende Annahme - wandeln sich. Und diesen Wandel, der eng mit dem generationalen Wandel verknüpft ist, empirisch zutreffend zu erfassen und theoretisch einzuordnen und zu erklären, ließe sich schließlich als eine wichtige Aufgabe erziehungswissenschaftlicher Geschlechterforschung begreifen. Möglich wäre es vielleicht dann, über die Diagnosen einer Verschiebung der gesellschaftlichen

9 Honig (1999: 214) bezeichnet Kind/Erwachsener sogar als die pädagogische Differenz.

10 So beispielsweise für den Zusammenhang von Gender Studies und Kindheitsforschung von Heinzel (2004: 172) oder für die erziehungswissenschaftliche Intersektionalitätsforschung als Kritik an der unzureichend theoretisierten Kategorie Alter von Kelle (2008: 57). 
Relevanz von Geschlecht auf latente Ebenen (Rendtorff 2015) oder einer Aktualisierung der Geschlechterdifferenz im Vexierspiel von gesellschaftlicher Vorder- und Hinterbühne (Baader 2015) hinaus, die Relevanz von Geschlecht und Geschlechterdifferenzierung auch vor dem Hintergrund differenter generationaler Erfahrungen einzuordnen.

Denn das, was uns als rhetorische Modernisierung (Wetterer 2003; Koppetsch/Burkart 1999) einer nach wie vor geschlechterhierarchisch strukturierten Ordnung erscheint - und erscheinen kann! - ist schließlich für jüngere Generationen, beispielsweise für Kinder, die mit geschlechterdifferenten Mathebüchern, rosafarbenen Überraschungseiern, Körperkorrekturen und Bodymodifikationen sowie multiplen Möglichkeiten geschlechtlicher und sexueller Identität aufwachsen, die Wirklichkeit, über die sie verfügen (können). Die in dieser Wirklichkeit liegenden Probleme und Chancen, die Kinder und Jugendliche betreffen und angehen, gilt es durch erziehungswissenschaftliche Forschung aufzuklären. Zugleich ließen sich damit auch die Handlungsprobleme der Erziehendengeneration mit kindlichen Geschlechterstereotypen und jugendlichen Geschlechterinszenierungen klarer fassen und auf Basis geschlechterreflexiver Pädagogik bearbeiten.

Welchen theoretisch-methodologischen Herausforderungen eine solche Analyse von ,modernisierten ' institutionellen Mechanismen, die jüngeren Generationen ,modernisierte " Plausibilitätsfiguren für die je zeithistorisch spezifizierte Form der Geschlechterdifferenzierung zur Verfügung stehen, ausgesetzt ist, wurde im vorliegenden Beitrag im Rückgriff auf die Gebundenheit der Forschenden an die konjunktiven Erfahrungsräume ihrer eigenen Generation hoffentlich schon etwas aufgeklärt. Eine empirische Konkretisierung der Analyseperspektive in Forschungsdesigns, die sich diesen Herausforderungen stellen, verspricht für eine sich als erziehungswissenschaftlich verstehende Geschlechterforschung zumindest interessante Erkenntnisse.

\section{Literaturverzeichnis}

AG Frauenforschung in der Erziehungswissenschaft in der Deutschen Gesellschaft für Erziehungswissenschaft (1991): Antrag an die Deutsche Gesellschaft für Erziehungswissenschaft auf Einrichtung einer Kommission „Frauenforschung in der Erziehungswissenschaft", S. 1-4, https://www.dgfe.de/fileadmin/OrdnerRedakteure/Sektionen/Sek11_FuGFiEW/1991_Antrag.pdf. [Zugriff: September 2018]

Baader, Meike Sophia (2015): Erziehung, Bildung, Geschlecht und Wissenschaft - Vexierspiele, De-Thematisierung, Hidden Gender Structures und Verschiebungen in einem komplexen Verhältnis. In: Walgenbach, Katharina/Stach, Anna (Hrsg.): Geschlecht in gesellschaftlichen Transformationsprozessen. Opladen/Berlin/Toronto: Barbara Budrich, S. 159-176. 
Baader, Meike Sophia (2016): History and gender matters. Erziehung - Gewalt - Sexualität in der Moderne in geschlechtergeschichtlicher Perspektive. In: Mahs, Claudia/Rendtorff, Barbara/Rieske, Thomas Viola (Hrsg.): Erziehung, Gewalt, Sexualität. Zum Verhältnis von Geschlecht und Gewalt in Erziehung und Bildung. Opladen/Berlin/Toronto: Barbara Budrich, S. 13-36.

Baumert, Jürgen/Tillmann, Klaus-Jürgen (2016): Empirische Bildungsforschung. Der kritische Blick und die Antwort auf die Kritiker. In: Zeitschrift für Erziehungswissenschaft, Sonderheft 31. Wiesbaden: Springer Verlag.

Benner, Dietrich (1980): Das Theorie-Praxis-Problem in der Erziehungswissenschaft und die Frage nach Prinzipien pädagogischen Denkens und Handelns. In: Zeitschrift für Pädagogik, 26, 4, S. 486-497.

Bohnsack, Ralf/Nentwig-Gesemann, Iris/Nohl, Arnd-Michael (Hrsg.) (2001): Die dokumentarische Methode und ihre Forschungspraxis. Grundlagen qualitativer Sozialforschung. Opladen: Leske \& Budrich. https://doi.org/10.1007/978-3-322-92213-7

Buchen, Sylvia/Helfferich, Cornelia/Maier, Maja S. (Hrsg.) (2004): Gender methodologisch. Empirische Forschung in der Informationsgesellschaft vor neuen Herausforderungen. Wiesbaden: Springer VS.

Casale, Rita/Rendtorff, Barbara (Hrsg.) (2018): Was kommt nach der Genderforschung? Zur Zukunft der feministischen Theoriebildung. Bielefeld: transcript.

Dinkelaker, Jörg/Meseth, Wolfgang/Neumann, Sascha/Rabenstein, Kerstin (2016): Die Erziehungswissenschaft, ihr Gegenstand und ihre Empirie. Sondierungen im Spannungsfeld von traditionellen Kontroversen und reflexiver Empirisierung. In: Meseth, Wolfgang/Dinkelaker, Jörg/Neumann, Sascha/Rabenstein, Kerstin/Dörner, Olaf/Hummrich, Merle/Kunze, Katharina (Hrsg.): Empirie des Pädagogischen und Empirie der Erziehungswissenschaft. Beobachtungen erziehungswissenschaftlicher Forschung. Bad Heilbrunn: Klinkhardt, S. 13-30.

Emmerich, Marcus/Hormel, Ulrike (2013): Heterogenität - Diversity - Intersektionalität. Zur Logik sozialer Unterscheidungen in pädagogischen Semantiken der Differenz. Wiesbaden: Springer.

Goffman, Erving (1994): Interaktion und Geschlecht. Frankfurt a. M.: Campus.

Grunert, Cathleen/Ludwig, Katja (2016): Labels matter!? Erziehungswissenschaftliche Studiengänge in der Bologna-Reform. In: Bildung und Erziehung, 69, S. 449-466.

Haraway, Donna (1996): Situiertes Wissen. Die Wissenschaftsfrage im Feminismus und das Privileg einer partialen Perspektive. In: Scheich, Elvira (Hrsg.): Vermittelte Weiblichkeit. Feministische Wissenschafts- und Gesellschaftstheorie. Hamburg: Brosch, S. 347-389.

Heinemann, Alisha. M. B./Mecheril, Paul (2016): Erziehungswissenschaftliche Diskriminierungsforschung. In: Scherr, Albert/El-Mafaalani, Aladin/Yüksel, Gökçen (Hrsg.): Handbuch Diskriminierung. Wiesbaden: Springer VS, S. 117-132. https://doi.org/10.1007/978-3-658-11119-9_6-1

Heinzel, Friederike (2004): Generationentheorien und erziehungswissenschaftliche Frauen- und Geschlechterforschung. In: Glaser, Edith/Klika, Dorle/Prengel, Annedore (Hrsg.): Handbuch Gender und Erziehungswissenschaft. Bad Heilbrunn: Klinkhardt, S. 157-174. 
Helsper, Werner (2016): Wird die Pluralität in der Erziehungswissenschaft aufgekündigt? In: Baumert, Jürgen/Tillmann, Klaus-Jürgen (Hrsg.): Empirische Bildungsforschung. Der kritische Blick und die Antwort auf die Kritiker. Zeitschrift für Erziehungswissenschaft, Sonderheft 31, S. 89-105.

Hirschauer, Stefan (2014): Wozu Gender Studies? Ein Forschungsfeld zwischen Feminismus und Kulturwissenschaft. In: Forschung \& Lehre. Alles, was die Wissenschaft bewegt, 11, S. 880-882.

Honig, Michael-Sebastian (1999): Entwurf einer Theorie der Kindheit. Frankfurt a. M.: Suhrkamp.

Jacoby, Juliane (2008): Die Erziehungswissenschaft im Jahr 2007. Potential und Grenzen feministischer Wissenschaftskritik in einer »handlungsorientierten « Wissenschaft. In: Casale, Rita/Rendtorff, Barbara (Hrsg.): Was kommt nach der Genderforschung? Zur Zukunft der feministischen Theoriebildung. Bielefeld: transcript, S. 83-100. https://doi.org/10.14361/9783839407486-007

Kelle, Helga (2008): Kommentar zum Beitrag: »Intersectionality« - ein neues Paradigma der Geschlechterforschung? In: Casale, Rita/Rendtorff, Barbara (Hrsg.): Was kommt nach der Genderforschung? Zur Zukunft der feministischen Theoriebildung. Bielefeld: transcript, S. 55-58.

Koller, Hans-Christoph/Wulftange, Gereon (Hrsg.) (2014): Lebensgeschichte als Bildungsprozess? Perspektiven bildungstheoretischer Biographieforschung. Bielefeld: trancript.

Koller, Hans-Christoph (2018): Erziehungswissenschaft. Mitteilungen der Deutschen Gesellschaft für Erziehungswissenschaft, 29, Opladen/Berlin/Toronto: Barbara Budrich.

König, Eckard/Zedler, Peter (1983): Einführung in die Wissenschaftstheorie der Erziehungswissenschaft. Düsseldorf: Schwann.

Koppetsch, Cornelia/Burkart, Günter (1999): Die Illusion der Emanzipation. Zur Wirksamkeit latenter Geschlechtsnormen im Milieuvergleich. Konstanz: Universitätsverlag Konstanz.

Ludwig, Katja (2018): „Es war ein Ringen“ - Zum Umgang mit disziplinärer Heterogenität im Kontext der Entwicklung erziehungswissenschaftlicher Hauptfachstudiengänge. In: Zeitschrift für Erziehungswissenschaft, S. 1-19, https://doi.org/10.1007/s11618-018-0845-8

Mannheim, Karl (1928): Das Problem der Generationen. In: Kölner Zeitschrift für Soziologie und Sozialpsychologie, 7, , S. 157-185.

Matthes, Eva (2018): Familie und Familienforschung in der Erziehungswissenschaft. In: Wonneberger, Astrid/Weidtmann, Katja/Stelzig-Willutzki, Sabina (Hrsg.): Familienwissenschaft. Grundlagen und Überblick. Wiesbaden: Springer VS, S. 249280. https://doi.org/10.1007/978-3-658-17003-5 10

Mollenhauer, Klaus (1980): Einige erziehungswissenschaftliche Probleme im Zusammenhang der Erforschung von „Alltagswelten Jugendlicher“. In: Lenzen, Dieter (Hrsg.): Pädagogik und Alltag. Methoden und Ergebnisse alltagsorientierter Forschung in der Erziehungswissenschaft. Stuttgart: Klett-Cotta, S. 97-111.

Müller, Hans-Rüdiger/Miethe, Ingrid (Hrsg.) (2012): Qualitative Bildungsforschung und Bildungstheorie. Opladen/Berlin/Toronto: Barbara Budrich.

Opitz, Claudia (2008): Nach der Gender-Forschung ist vor der Genderforschung. Plädoyer für die historische Perspektive in der Geschlechterforschung. In: Casale, 
Rita/Rendtorff, Barbara (Hrsg.): Was kommt nach der Genderforschung? Zur Zukunft der feministischen Theoriebildung. Bielefeld: transcript, S. 13-28.

Pfaff, Nicolle (2015): Erziehungswissenschaftliche Jugendforschung am Neubeginn? Aktuelle Gegenstandsfelder und Perspektiven in der Forschung zu Jugend. In: Sandring, Sabine/Helsper, Werner/Krüger, Heinz-Hermann (Hrsg.): Jugend. Theoriediskurse und Forschungsfelder. Wiesbaden: Springer VS, S. 35-55.

Rendtorff, Barbara (2015): Zugewinne und Fallen - aktuelle Veränderungen in Geschlechtervorstellungen und ihre Probleme. In: Dausien, Bettina/Thon, Christine/Walgenbach, Katharina (Hrsg.): Geschlecht - Sozialisation - Transformationen. Opladen/Berlin/Toronto: Barbara Budrich, S. 77- 92.

https://doi.org/10.3224/jfgfe.v11i1.04

Rieger-Ladich (2009): Konturen einer machtkritischen Disziplingeschichte: Methodologische Überlegungen und erste Befunde für die erziehungswissenschaftliche Geschlechterforschung. In: Andresen, Sabine/Glaser, Edith (Hrsg.): Disziplingeschichte der Erziehungswissenschaft als Geschlechtergeschichte. Jahrbuch Frauen- und Geschlechterforschung in der Erziehungswissenschaft 5, Opladen: Barbara Budrich, S. 15-27.

Schmid, Pia (2008): Kommentar zum Beitrag: Nach der Gender-Forschung ist vor der Genderforschung. In Casale, Rita/Rendtorff, Barbara (Hrsg.): Was kommt nach der Genderforschung? Zur Zukunft der feministischen Theoriebildung. Bielefeld: transcript, S. 29-32.

Sektion Frauen- und Geschlechterforschung in der Erziehungswissenschaft (o.J.): Archiv, https://www.dgfe.de/sektionen-kommissionen/sektion-11-frauen-und-inder-erziehungswissenschaft/archiv.html. [Zugriff: September 2018].

Terhart, Ewald (2012): „Bildungswissenschaften“. Verlegenheitslösung, Sammeldisziplin, Kampfbegriff? In: Zeitschrift für Pädagogik, 58, S. 22-39.

Terhart, Ewald (2016): Empirische Bildungsforschung und ihre Disziplinen - Wandlungsprozesse und Konfliktlinien in instabilen Expertenkulturen. In: Baumert, Jürgen/Tillmann, Klaus-Jürgen (Hrsg.): Empirische Bildungsforschung. Der kritische Blick und die Antwort auf die Kritiker. Zeitschrift für Erziehungswissenschaft, Sonderheft 31, S. 73-87. https://doi.org/10.1007/s11618-016-0708-0

Vogel, Peter (2016): Die Erziehungswissenschaft und ihr Wissen. Selbstkritik, Thematisierungsformen, Analytik. In: Zeitschrift für Pädagogik, 62, S. 452-473.

Völker, Susanne (2017): „Herausforderungen der Geschlechterforschung“. Eröffnung der gemeinsamen Konferenz der D-A-CH Fachgesellschaften für Geschlechterforschung/-studien vom 28.-30.09.2017 in Köln, S. 1-6. https://www.fg-gender.de/wp-content/uploads/2017/10/Voelker_Herausforderungen-Geschlechterforschung-28092917-2.pdf. [Zugriff: September 2018].

Von Rosenberg, Florian (2011): Bildung und Habitustransformation. Empirische Rekonstruktionen und bildungstheoretische Reflexionen. Bielefeld: transcript.

Vorstand der Sektion Frauen- und Geschlechterforschung in der DGfE (2013): Rundbrief August 2013, S. 1-43. https://www.dgfe.de/fileadmin/OrdnerRedakteure/Sektionen/Sek11_FuGFiEW/Rundbrief_08_2013.pdf. [Zugriff: September 2018].

Walgenbach, Katharina (2014): Heterogenität, Intersektionalität, Diversity in der Erziehungswissenschaft. Opladen: Barbara Budrich.

Wetterer, Angelika (2003): Rhetorische Modernisierung. Das Verschwinden der Ungleichheit aus dem zeitgenössischen Differenzwissen. In: Knapp, GudrunAxeli/Wetterer, Angelika (Hrsg.): Achsen der Differenz. Gesellschaftstheorie und 
feministische Kritik 2. Forum Frauenforschung, Band 16, Münster: Westfälisches Dampfboot, S. 286-319.

Zedler, Peter (2016): Allgemeine Erziehungswissenschaft und Empirische Bildungsforschung. Entwicklungslinien eines gelegentlich schwierigen Verhältnisses. Teil 1 und Teil 2. In: Fickermann, Detlef/Fuchs, Hans-Werner (Hrsg.): Bildungsforschung - disziplinäre Zugänge: Fragestellungen, Methoden und Ergebnisse. Münster/New York: Waxmann, S. 69-101. 